BULGARIAN ACADEMY OF SCIENCES

CYBERNETICS AND INFORMATION TECHNOLOGIES • Volume 15, No 6

Special Issue on Logistics, Informatics and Service Science

Sofia $\bullet 2015$

Print ISSN: 1311-9702; Online ISSN: 1314-4081

DOI: $10.1515 /$ cait-2015-0063

\title{
2.5D Sound Field Reproduction Using Higher Order Loudspeakers
}

\section{Maoshen Jia, Wenbei Wang, Ziyu Yang}

School of Electronic Information and Control Engineering, Beijing University of Technology, Beijing, 100124 China

Emails:jiamaoshen@bjut.edu.cn_wwb@emails.bjut.edu.cn_yangziyu@emails.bjut.edu.cn

Abstract: Using 3-Dimensional (3D) sound sources as secondary sources to 2-Dimensional (2D) sound field reproduction, it is termed 2.5-Dimensional (2.5D) sound field reproduction which is currently drawing broad interest in acoustic signal processing. In this paper we propose a method to reproduce a $2 D$ sound field, using a circular array of $3 \mathrm{D}$ High Order $(\mathrm{HO})$ loudspeakers, which provides a mode matching solution based on $3 D$ wave field translation. Using the spherical addition theorem, we first obtain a spherical harmonics representation of a $2 D$ sound field reproduced by an array of $\mathrm{HO}$ loudspeakers. Then, the corresponding relationship between the reproduced sound field and the desired sound field is established by spherical/cylindrical harmonic expansions. Finally, the modal weights of HO loudspeakers are designed by using a least squares method. Simulation results show that the proposed method extends the reproduction region and significantly reduces the required minimum number of loudspeakers over the other referenced methods.

Keywords: $2.5 D$ reproduction, sound field, high order loudspeaker, circular loudspeaker array.

\section{Introduction}

Spatial sound field reproduction is a fundamental topic in acoustic signal processing. The aim of the sound field reproduction is to reproduce a desired sound field in a given region of space. Using an array of loudspeakers, the sound field 
reproduction system can give listeners the impression of being immersed in a realistic, yet virtual sound environment. According to psychoacoustic theory, the human ear is sensitive to the horizontal sound field at the same height. Hence, the reproduction technique of 2-Dimensional (2D), or height invariant, sound fields is more efficient in practical situations. In the existing commercial sound field reproduction systems, such as Dolby Surround [1], all loudspeakers are positioned in the horizontal plane at a height approximately to the level of the listener's ears.

In earlier studies of $2 \mathrm{D}$ sound field reproduction, line sources that exhibit $2 \mathrm{D}$ transfer functions [2-4] were theoretically employed as secondary sources for reproduction. However, the spatial properties of the actual loudspeakers can be more accurately modelled by 3-Dimensional (3D) sound sources or loudspeakers rather than line sources. Using 3D sound sources (or loudspeakers) as secondary sources, the $2 \mathrm{D}$ sound field reproduction has been termed as 2.5-Dimensional (2.5D) sound field reproduction [5].

Several techniques of $2.5 \mathrm{D}$ sound field reproduction have already been proposed, which are based on distinct representations of a sound field. One technique is the Spectral Division Method (SDM) [6,7], which establishes the 2.5D reproduction equation in a wave number domain with the employment of linear distribution of secondary sources. Another technique is the Wave Field Synthesis (WFS)-based method [3,5], in which two separate stationary phase approximations are employed by using the Kirchhoff-Helmholtz integral equation. The reproduction is accurate only for large distances of the virtual source or the listener's location to the secondary source positions $[2,5]$. Meanwhile, the aperture between the loudspeakers is limited by the spatial sampling process [8]. However, one characteristic in 2.5D WFS and SDM reproduction is that large numbers of loudspeakers are required for reproduction over large areas. Therefore, Higher Order Ambisonics (HOA) has been related to the implementation of 2.5D sound field reproduction recently [9], where HOA is based on the cylindrical/spherical harmonic representations of a sound field. In this method, 3D point sources are employed as secondary sources, which provide a continuous the loudspeaker concept solution in a reproduction model. Therefore, better reproduction accuracy can be achieved. Implementation of $2.5 \mathrm{D} \mathrm{HOA}$ has practical significance in combination with the development of the spatial sound. However, for high frequencies over large areas in 2.5D HOA, a great number of loudspeakers is required, as minimum, in order to accurately reproduce a height invariant sound field. In addition, in a 2.5D HOA system, the radius of the reproduction region is smaller than that of the loudspeakers array region, which results in waste of space.

To address the above issues, since the High Order (HO) loudspeakers can both radiate sound with multiple radiation patterns [10-12] and achieve better improvements for sound field reproduction than monopole loudspeakers, i.e., point sources, we apply 3D HO loudspeakers to $2.5 \mathrm{D}$ sound field reproduction. In this paper, based on spherical harmonic expansions, a method is proposed to reproduce 2D or height invariant sound fields with a circular array of HO loudspeakers. Firstly, spherical harmonics representation of 2D sound field reproduced, by an array of HO loudspeakers is obtained, based on the spherical addition theorem. 
Secondly, the corresponding relationship between the reproduced sound field and the desired sound field is established by spherical/cylindrical harmonic expansions. Finally, the modal weights of HO loudspeakers are designed by using the least squares method. Simulation results show that the method proposed outperforms the other referenced methods

The remainder of this paper is organized as follows. Section 2 presents a detailed description for the decomposition of the desired sound field using cylindrical harmonics. Section 3 introduces the proposed 2.5D sound field reproduction approach. Simulation results are presented in Section 4.

\section{Cylindrical harmonics analysis of the desired sound field}

This paper mainly concentrates on the reproduction of $2 \mathrm{D}$ or height invariant sound fields which are considered as desired sound fields. At physical level, the 2D sound field can be represented by acoustic pressure within a given region of space. In this section the cylindrical harmonic analysis [3] for the desired sound field will be discussed.

In cylindrical coordinates, propagation of acoustic pressure perturbations $p_{d}(r, \phi, z, t)$ in a homogeneous medium is represented by the wave equation, which is defined as [13]:

$$
\nabla^{2} p_{d}(r, \phi, z, t)-\frac{1}{c^{2}} \frac{\partial^{2} p_{d}(r, \phi, z, t)}{\partial t^{2}}=0,
$$

where $t, r, \phi$ and $z$ represent the time index, radius, azimuth and height of an arbitrary observation point, respectively; $c=340 \mathrm{~m} / \mathrm{s}$ is the speed of sound propagation and $\nabla^{2}$ is the Laplace operator. After converting the time domain wave equation to the frequency domain, the representation of a $2 \mathrm{D}$, height invariant, sound field can be written in the following form via separation of the variables [5]:

$$
P_{d}(\boldsymbol{x}, k)=\sum_{m=-\infty}^{\infty} \alpha_{m}(k) J_{m}(k r) e^{i m \phi},
$$

where $P_{d}(\boldsymbol{x}, k)$ denotes the acoustic pressure of a given sound field at an observation point $\boldsymbol{x}=(r, \phi) ; k$ is the wave number satisfied $k=2 \pi f / c$, where $f$ is the frequency and $c$ is the speed of sound; $\alpha_{m}(k)$ are a set of harmonic expansion coefficients, and $J_{m}(\cdot)$ is the $m$-th order Bessel function of the first kind. For a 2D wave source of a unit strength located at $\boldsymbol{y}_{v}=\left(r_{v}, \phi_{v}\right)$ (where $r_{v}>r$ ), based on the Jacobi-Anger expression and the addition property of the Hankel function [3], the expansion coefficients $\alpha_{m}(k)$ of a plane wave or a cylindrical wave can be derived as follows:

$$
\alpha_{m}(k)=\left\{\begin{array}{cl}
i^{m} e^{-i m \phi_{v}}, & \text { plane wave, } \\
\frac{i}{4} H_{m}^{(2)}\left(k r_{v}\right) \mathrm{e}^{-i m \phi_{v}}, & \text { cylindrical wave, }
\end{array}\right.
$$

where $H_{m}{ }^{(2)}(\cdot)$ is the $m$-th order Hankel function of the second kind. The representation (2) has an infinite number of orthogonal modes, which is not possible to be achieved. Therefore, due to the properties of the Bessel function, we can 
truncate this series expansion to a finite number within the region of interest, i.e., a region within a circle of radius $r_{l}\left(\forall r \leq r_{l}\right)$. Hence, (2) can be truncated to $M$ terms as:

$$
P_{d}(\boldsymbol{x}, k)=\sum_{m=-M}^{M} \alpha_{m}(k) J_{m}(k r) e^{i m \phi},
$$

where the truncation order $M=\left\lceil e k r_{l} / 2\right\rceil, J_{m}(k r) \mathrm{e}^{i m \phi}$ is the basis function of cylindrical harmonic expansion.

From (4) it can be observed that a desired 2D sound field can be determined by $2 M+1$ cylindrical harmonic coefficients. Therefore, the key issue of the sound field reproduction is to use loudspeakers to describe the expansion coefficients $\alpha_{m}(k)$.

\section{Proposed method of $2.5 \mathrm{D}$ sound field reproduction}

In practical applications, considering the cost of equipment and the complexity of design, we always employ a limited number of loudspeakers in the sound field reproduction systems. To avoid spatial aliasing [14] which is caused by the discretization processing of the continuous loudspeakers distribution, the minimum required number of the monopole loudspeakers $Q$ should be satisfied with the condition $Q \geq 2 M+1$. Hence, a large number of loudspeakers is needed for the reproduction of high frequencies over significant areas. According to the properties of HO loudspeakers $[11,12,15]$, it can be observed that HO loudspeakers can radiate a sound with multiple radiation patterns. Using such HO loudspeakers, we can obtain further improvements in sound field reproduction than monopole loudspeakers. Meanwhile, the array of loudspeakers made up of HO loudspeakers occupies a smaller space.

Based on the above advantages, HO loudspeakers are introduced to $2.5 \mathrm{D}$ sound field reproduction in this section, where in subsection 3.1, a spherical harmonics representation of 2D sound field reproduced by an array of $\mathrm{HO}$ loudspeakers is obtained. Then, the modal weights of HO loudspeakers are designed in subsection 3.2.

\subsection{Spherical harmonics representation of the reproduction sound field}

In the spherical coordinates, given an observation point $x=(r, \theta, \phi)$, a $3 \mathrm{D} \mathrm{HO}$ loudspeaker positioned at $\boldsymbol{x}_{l}=\left(r_{l}, \theta_{l}, \phi_{l}\right)$ of a circular region in the horizontal plane will radiate a sound field as given below:

$$
P_{l}(\boldsymbol{x}, k)=e^{-i \omega t} \sum_{\hat{n}=0}^{\hat{N}} \sum_{\hat{m}=-\hat{n}}^{\hat{n}} \frac{w_{\hat{n}, \hat{m}}(k)}{h_{\hat{n}}^{\prime}\left(k r_{0}\right)} h_{\hat{n}}\left(k\left\|\boldsymbol{x}-\boldsymbol{x}_{l}\right\|\right) Y_{\hat{n}, \hat{m}}\left(\hat{\boldsymbol{y}}_{l}\right),
$$

where $r, \theta$ and $\phi$ denote the radius, elevation and azimuth, respectively; $P_{l}(\boldsymbol{x}, k)$ is the acoustic pressure at the observation point $\boldsymbol{x} ; \hat{N}$ is the order of HO loudspeaker, $w_{\hat{n}, \hat{m}}(k)$ are complex amplitudes which represent the modal weights; $h_{\hat{n}}^{\prime}\left(k r_{0}\right)$ denotes the first-order derivative of the spherical Hankel function of the first kind, $r_{0}$ is the radius of the HO loudspeaker; $e^{-i \omega t}$ is the time factor with respect to the 
angular velocity $\omega$ and the time $t$ (only static situation $t=0$ is considered), and $\|\cdot\|$ represents the vector 2-norm. Due to the fact that $\boldsymbol{x}_{l}$ and $\boldsymbol{x}$ are in the same horizontal plane, the elevation of $\boldsymbol{x}$ equals to that of $\boldsymbol{x}_{l}$ in the spherical coordinates, which is $\pi / 2 ; \hat{y}_{l}=\left(\pi / 2, \bar{\phi}_{l}\right)$ is the direction vector of $\boldsymbol{y}_{l}$ and $\boldsymbol{y}_{l}=\boldsymbol{x}-\boldsymbol{x}_{l}$.

The spherical harmonics can be defined as [14]:

$$
Y_{\hat{n}, \hat{m}}\left(\hat{\boldsymbol{y}}_{l}\right)=(-1)^{\hat{m}} A_{\hat{n}, \hat{m}} P_{\hat{n}, \hat{m} \mid}(\cos (\pi / 2)) E_{\hat{m}}(\bar{\phi}),
$$

where $P_{\hat{n},|\hat{m}|}(\cdot)$ is the associated Legendre function. Note that $E_{\hat{m}}\left(\bar{\phi}_{l}\right)=\mathrm{e}^{\mathrm{i} \dot{m} \overline{\phi_{l}}}$ holds the orthogonality property. $A_{\hat{n}, \hat{m}}$ is defined as follows:

$$
A_{\hat{n}, \hat{m}}=\sqrt{\frac{(2 \hat{n}+1)}{4 \pi} \frac{(\hat{n}-|\hat{m}|) !}{(\hat{n}+|\hat{m}|) !}} .
$$

From (5) we can find that the 3D polar patterns of the spherical harmonics carry the directional information, and thus not all polar patterns of the weighted spherical harmonics are on the horizontal plane. Only when $\hat{m}=-\hat{n}$ and $\hat{m}=\hat{n}$, the response to the $3 \mathrm{D}$ HO loudspeakers will have a contribution to the reproduction of the $2 \mathrm{D}$ sound field. Then we can rewrite (5) as

$$
\begin{aligned}
P_{l}(\boldsymbol{x}, k) & =\sum_{\hat{n}=0}^{\hat{N}} \sum_{\hat{m}=\{-\hat{n}, \hat{n}\}} \frac{w_{\hat{n}, \hat{m}}(k)}{h_{\hat{n}}^{\prime}\left(k r_{0}\right)} h_{\hat{n}}\left(k\left\|\boldsymbol{x}-\boldsymbol{x}_{l}\right\|\right) Y_{\hat{n}, \hat{m}}\left(\hat{\boldsymbol{y}}_{l}\right)= \\
& =\sum_{\hat{m}=-\hat{N}}^{\hat{N}} \sum_{\hat{n}=\hat{n} \mid} \frac{w_{\hat{n}, \hat{m}}(k)}{h_{\hat{n}}^{\prime}\left(k r_{0}\right)} h_{\hat{n}}\left(k\left\|\boldsymbol{x}-\boldsymbol{x}_{l}\right\|\right) Y_{\hat{n}, \hat{m}}\left(\hat{\boldsymbol{y}}_{l}\right) .
\end{aligned}
$$

We employ $L$ number of such HO loudspeakers in a circular discrete array, where the $l$-th $(l=1,2, \ldots, L)$ loudspeaker is located at $\boldsymbol{x}_{l}=\left(r_{l}, \phi_{l}\right)$. Therefore, the sound field can be represented as

$$
P_{r}(\boldsymbol{x}, k)=\sum_{l=1}^{L} P_{l}(\boldsymbol{x}, k)=\sum_{l=1}^{L} \sum_{\hat{m}=-\hat{N}}^{\hat{N}} \sum_{\hat{n}=|\hat{\hat{m}}|} \frac{w_{\hat{n}, \hat{m}}^{(l)}(k)}{h_{\hat{n}}^{\prime}\left(k r_{0}\right)} h_{\hat{n}}\left(k\left\|\boldsymbol{x}-\boldsymbol{x}_{l}\right\|\right) Y_{\hat{n}, \hat{m}}\left(\hat{\boldsymbol{y}}_{l}\right), \quad\left(r \leq r_{l}\right) .
$$

According to the spherical addition theorem for the spherical Hankel function [16], $h_{\hat{n}}\left(k\left\|\boldsymbol{x}-\boldsymbol{x}_{l}\right\|\right) Y_{\hat{n}, \hat{m}}\left(\hat{\boldsymbol{y}}_{l}\right)$ can be represented as

$$
h_{\hat{n}}\left(k\left\|\boldsymbol{x}-\boldsymbol{x}_{l}\right\|\right) Y_{\hat{n}, \hat{m}}\left(\hat{\boldsymbol{y}}_{l}\right)=\sum_{n=0}^{\infty} \sum_{m=-n}^{n} S_{\hat{n}, n}^{\hat{m}, m}\left(\boldsymbol{x}_{l}\right) j_{n}(k r) Y_{n, m}(\hat{\boldsymbol{x}}),
$$

where $\hat{\boldsymbol{x}}=(\pi / 2, \phi)$ is the direction vector of $\boldsymbol{x}, j_{n}(\cdot)$ is the first kind spherical Bessel function of $n$-th degree.

By substituting (10) with (9), we have

$$
P_{r}(\boldsymbol{x}, k)=\sum_{n=0}^{\infty} \sum_{m=-n}^{n}\left\{j_{n}(k r) Y_{n, m}(\hat{\boldsymbol{x}}) \sum_{l=1}^{L} \sum_{\hat{m}=-\hat{N}}^{\hat{N}} \sum_{\hat{n}=|\hat{m}|} \frac{w_{\hat{n}, \hat{m}}^{(l)}(k)}{h_{\hat{n}}^{\prime}\left(k r_{0}\right)} S_{\hat{n}, n}^{\hat{m}, m}\left(\boldsymbol{x}_{l}\right)\right\},
$$

where

(12) $S_{\hat{n}, n}^{\hat{n}, m}\left(\boldsymbol{x}_{l}\right)=i^{(n-\hat{n}+4 \hat{m}-2 m)} \sum_{q=0}^{n+\hat{n}} i^{q} h_{q}\left(k r_{l}\right) Y_{q,(m-\hat{m})}^{*}\left(\hat{\boldsymbol{x}}_{l}\right) \sqrt{4 \pi(2 n+1)(2 \hat{n}+1)(2 q+1)} W_{1} W_{2}$, 
in which $\hat{\boldsymbol{x}}_{l}=\left(\pi / 2, \phi_{l}\right)$ is the direction vector of $\boldsymbol{x}_{l}$. The symbol * denotes the complex conjugate operation. $W_{1}, W_{2}$ denote Wigner $3-j$ symbols which are defined by

$$
W_{1}=\left(\begin{array}{ccc}
\hat{n} & n & q \\
0 & 0 & 0
\end{array}\right), \quad W_{2}=\left(\begin{array}{ccc}
\hat{n} & n & q \\
\hat{m} & -m & (m-\hat{m})
\end{array}\right) .
$$

By changing the summation order of (11), the sound field can be represented as

$$
P_{r}(\boldsymbol{x}, k)=\sum_{m=-\infty}^{\infty} \sum_{n=|m|}^{\infty}\left\{j_{n}(k r) Y_{n, m}(\hat{\boldsymbol{x}}) \sum_{l=1}^{L} \sum_{\hat{m}=-\hat{N}}^{\hat{N}} \sum_{\hat{n}=|\hat{m}|} \frac{w_{\hat{n}, \hat{m}}^{(l)}(k)}{h_{\hat{n}}^{\prime}\left(k r_{0}\right)} S_{\hat{n}, n}^{\hat{m}, m}\left(\boldsymbol{x}_{l}\right)\right\} .
$$

Due to the high pass nature of spherical Bessel functions [14], the infinite representation (13) can be replaced by a finite sum, and then substituting (6) with (13), we can obtain the sound field as follows:

(14) $P_{r}(\boldsymbol{x}, k)=\sum_{m=-M}^{M} \sum_{n=|m|}^{M} \sum_{l=1}^{L} \sum_{\hat{m}=-\hat{N}}^{\hat{N}} \sum_{n=|\hat{m}|}(-1)^{m} \frac{w_{\hat{n}, \hat{m}}^{(l)}(k)}{h_{\hat{n}}^{\prime}\left(k r_{0}\right)} S_{\hat{n}, n}^{\hat{m}, m}\left(\boldsymbol{x}_{l}\right) j_{n}(k r) A_{n, m} P_{n,|m|}(\cos (\pi / 2)) E_{m}(\phi)$,

where $M$ is the truncation order which denotes the number of the highest nonzero.

\section{2. $2.5 \mathrm{D}$ sound field reproduction}

The key issue in the $2.5 \mathrm{D}$ sound field reproduction is to design the modal weights of HO loudspeakers. Hence, this subsection will establish the corresponding relationship between the desired sound field and the sound field reproduced by (14).

Note that the summation over $n$ in (14) reduces it to a single term with $n=|m|$. If the modal weights $w_{\hat{n}, \hat{m}}^{(l)}(k)$ satisfy the condition that (14) equals to the expansion of (4), an array of $L \mathrm{HO}$ loudspeakers located in a circular region of radius $r_{l}$, can exactly reproduce the desired sound field at all observation points $r<r_{l}$. Therefore, by minimizing the least squares errors between the desired sound field and the reproduced field sound field, i.e., $\left|P_{r}(\boldsymbol{x}, k)-P_{d}(\boldsymbol{x}, k)\right|^{2}$, we have:

(15) $\sum_{n=|m|} \sum_{l=1}^{L} \sum_{\hat{m}=-\hat{N}}^{\hat{N}} \sum_{\hat{n}=|\hat{m}|}(-1)^{m} \frac{w_{\hat{n}, \hat{m}}^{(l)}(k)}{h_{\hat{n}}^{\prime}\left(k r_{0}\right)} S_{\hat{n}, n}^{\hat{n}, m}\left(\boldsymbol{x}_{l}\right) j_{n}(k r) A_{n, m} P_{n,|m|}(\cos (\pi / 2))=\alpha_{m}(k) J_{m}(k r)$.

In (15), given a certain $r$, a set of modal weights $w_{\hat{n}, \hat{m}}^{(l)}(k)$ can be achieved. To guarantee the minimum reproduction error and obtain the optimal modal weights, let $r$ tends to zero [17], and then we have

$$
j_{n}(k r) \approx \frac{(k r)^{n}}{(2 n+1) ! !}, \quad J_{m}(k r) \approx \frac{\left(\operatorname{sgn}(m)^{|m|}\right)(k r)^{|m|}}{2^{|m|}|m| !} .
$$

According to the application of L'Hôpital's theorem [18], we can obtain

$$
C_{m}=\lim _{r \rightarrow 0} \frac{j_{n}(k r)}{J_{m}(k r)}=\frac{2^{|m|}|m| !}{\left(\operatorname{sgn}(m)^{|m|}\right)(2|m|+1) ! !},
$$

where $\operatorname{sgn}(\cdot)$ represents the sign function. By substituting (16) and (17) with (15), we can obtain 


$$
\sum_{n=|m|} \sum_{l=1}^{L} \sum_{\hat{m}=-\hat{N}}^{\hat{N}} \sum_{\hat{n}=|\hat{m}|}(-1)^{m} \frac{w_{\hat{n}, \hat{m}}^{(l)}(k)}{h_{\hat{n}}^{\prime}\left(k r_{0}\right)} S_{\hat{n}, n}^{\hat{m}, m}\left(\boldsymbol{x}_{l}\right) A_{n, m} P_{n,|m|}(\cos (\pi / 2)) C_{m}=\alpha_{m}(k) .
$$

According to the theorem of matrix multiplication, (18) can be represented as

$$
\boldsymbol{H} \boldsymbol{A}=\boldsymbol{\alpha},
$$

where $\boldsymbol{A}=\left[\boldsymbol{G}^{(1)}, \boldsymbol{G}^{(2)}, \ldots, \boldsymbol{G}^{(L)}\right]^{\mathrm{T}}$ is a $[L(2 \hat{N}+1) \times 1]$ vector representing the modal weights of $\mathrm{HO}$ loudspeakers with $\boldsymbol{G}^{(l)}=\left[w_{\hat{N},-\hat{N}}^{(l)}(k), w_{\hat{N}-1,-\hat{N}+1}^{(l)}(k), \ldots, w_{\hat{N}, \hat{N}}^{(l)}(k)\right]^{\mathrm{T}}$. $\boldsymbol{\alpha}=\left[\alpha_{-M}(k), \alpha_{-M+1}(k), \ldots, \alpha_{M}(k)\right]^{\mathrm{T}}$ is a vector representing the desired field coefficients. The translation matrices $\boldsymbol{H}=\left[\boldsymbol{T}^{(1)}, \boldsymbol{T}^{(2)}, \ldots, \boldsymbol{T}^{(L)}\right], \boldsymbol{T}^{(l)}, l=1,2, \ldots, L$, are attained as follows:

$$
\boldsymbol{T}^{(l)}=\left[\begin{array}{ccccc}
T_{\hat{N}, M}^{-\hat{N},-M}(l) & T_{\hat{N}-1, M}^{-\hat{N}+1,-M}(l) & \cdots & \cdots & T_{\hat{N}, M}^{\hat{N},-M}(l) \\
\vdots & \vdots & \vdots & \vdots & \vdots \\
T_{\hat{N}, M}^{-\hat{N}, M}(l) & T_{\hat{N}-1, M}^{-\hat{N}+1, M}(l) & \cdots & \cdots & T_{\hat{N}, M}^{\hat{N}, M}(l)
\end{array}\right],
$$

where $T_{\hat{n}, n}^{\hat{m}, m}(l)=\frac{S_{\hat{n}, n}^{\hat{n}, m}\left(\boldsymbol{x}_{l}\right)}{h_{\hat{n}}^{\prime}\left(k r_{o}\right)} A_{n, m} P_{n,|m|}(\cos (\pi / 2)) C_{m}$. According to $\boldsymbol{H}$ and $\boldsymbol{\alpha}$, the required modal weights of HO loudspeakers can be obtained by

$$
\boldsymbol{A}=\boldsymbol{H}^{\#}\left[\boldsymbol{H} \boldsymbol{H}^{\#}+\gamma \boldsymbol{I}^{-1} \boldsymbol{\alpha},\right.
$$

where \# represents the Hermitian Transpose, and $\boldsymbol{I}$ is a $[(2 M+1) \times(2 M+1)]$ identity matrix. Note that $\gamma$ is the regularization control parameter and $\gamma=0$ indicates the minimum energy solution of (21).

To avoid spatial aliasing, the required number of HO loudspeakers $L$ of the proposed method needs to satisfy $L \cdot(2 \hat{N}+1) \geq(2 M+1)$. And in reference to [9], the number of monopole loudspeakers $Q$ for sound field reproduction meets $Q \geq(2 M+1)$. Hence, for a given frequency $f$ and a radius of the reproduced region $r_{l}$, the minimum required number of $\mathrm{HO}$ loudspeakers will be decreased by a factor $1 /(2 \hat{N}+1)$ compared to the monopole loudspeakers, i.e., $L=Q /(2 \hat{N}+1)$.

\section{Simulation results}

In order to evaluate the performance of the proposed scheme, three simulation experiments are considered in this section. Firstly, we test the performance of the proposed method in various orders of the HO loudspeakers. Secondly, the comparison experiment results are given over a referenced method. Finally, we evaluate the reproduction errors of the sound field for various frequencies. In these experiments, $L \mathrm{HO}$ loudspeakers are used, which are equally distributed in a circular array of radius $r_{l}=3 \mathrm{~m}$. The HOA method [9], based on point sources for $2.5 \mathrm{D}$ reproduction is employed as the referenced approach.

A) The first experiment: For convenience of the illustration purposes, a monochromatic plane wave and a cylindrical wave are chosen as desired sound sources. It should be noted that the proposed method is not limited to reproducing 
plane wave and cylindrical wave sound fields. We simulate a 2D plane wave and a cylindrical wave of frequency $1 \mathrm{kHz}$, deriving from $\phi_{v}=\pi / 4$ (and $r_{v}=4 \mathrm{~m}$ for the cylindrical wave source) in this experiment. This is equivalent to $k r_{l}=55.412$, and according to $M=\left\lceil e k r_{l} / 2\right\rceil$, we can get $M=76$. Thus the minimum number of HO loudspeakers is $L=31$. Simulations are carried out in three cases, i.e., $\hat{N}=2, \hat{N}=3$, and $\hat{N}=4$. The reproduced pressure fields are shown in Fig.1. These figures are calculated at $300 \times 300$ points and displayed as a "density plot", meaning that the acoustic pressures are represented by various shades of gray. The pressures greater than 1 are black, the pressures less than -1 are white, and the pressures between -1 and 1 are appropriately shaded. The HO loudspeakers are shown as " $\mathrm{x}$ " marks. From Fig. 1 it can be observed that the desired interior sound field is exactly reproduced within the array of HO loudspeakers. In addition, the reproduction errors in the exterior sound field distinctly decrease when increasing the order of loudspeakers. Furthermore, the radius of the reproduction sound field is close to $r_{l}=3 \mathrm{~m}$.
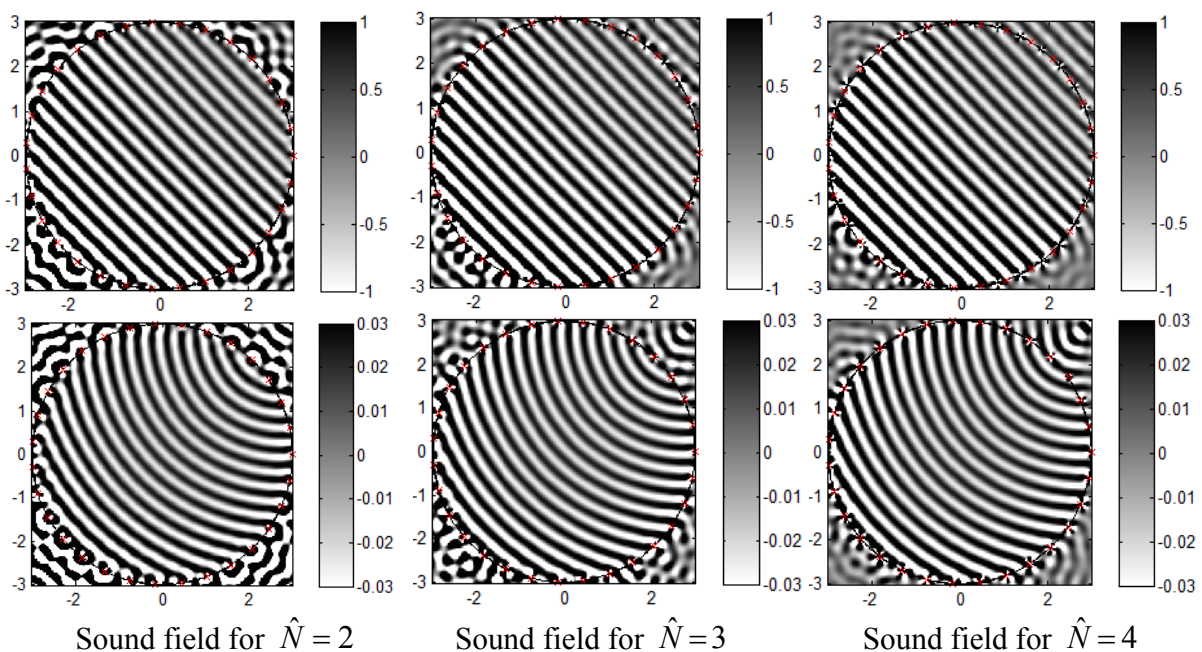

Fig. 1. Reproduction of a $2 \mathrm{D}$ plane wave from $\phi_{v}=\pi / 4$ (upper row) and a cylindrical wave from $\phi_{v}=\pi / 4, r_{v}=4 \mathrm{~m}$ (lower row) for different orders. The HO loudspeakers are equally placed on a circle of radius $3 \mathrm{~m}$. The HO loudspeakers are shown as " $\mathrm{x}$ " marks

B) The second experiment: A plane wave and a cylindrical wave deriving from $\phi_{v}=\pi / 4$ (and $r_{v}=4 \mathrm{~m}$ for cylindrical wave) are simulated by the proposed method and the referenced method, respectively. Considering that the reproduction error of the proposed method in the second order case (i.e., $\hat{N}=2$ ) is 0.0142 calculated by (22), it agrees well with the expected value of 0.04 in [3]. So the second order case was chosen for the evaluation comparison. In this experiment, 31 HO loudspeakers are required in the proposed scheme and 53 point sources (monopole loudspeakers) are used in the referenced method for $2.5 \mathrm{D}$ reproduction. The reproduced sound fields are shown in Fig. 2. From Fig. 2a and Fig. 2b it can be seen that the reproduced 2.5D sound field based on HO loudspeakers matches well 
with the desired field within the circle region. By comparing Fig. 2b and Fig. 2c, it can be observed that the area of the reproduction region of the proposed method is much larger than that of the referenced method (i.e., the interior circle in Fig. 2c) with the same radius of the loudspeaker array. Meanwhile, the number of the loudspeakers used in the proposed method is slightly fewer than in the referenced method.

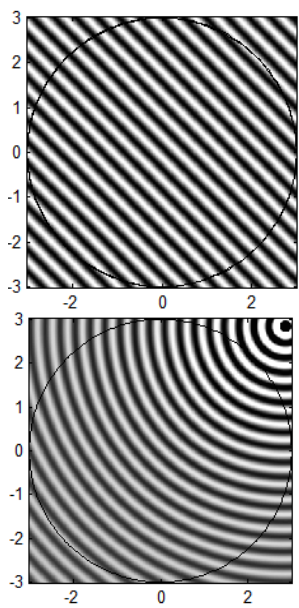

(a)
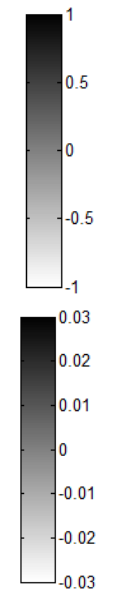

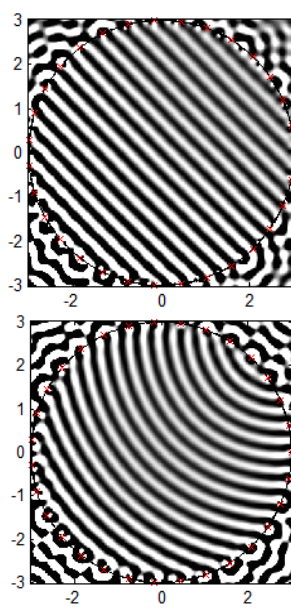

(b)
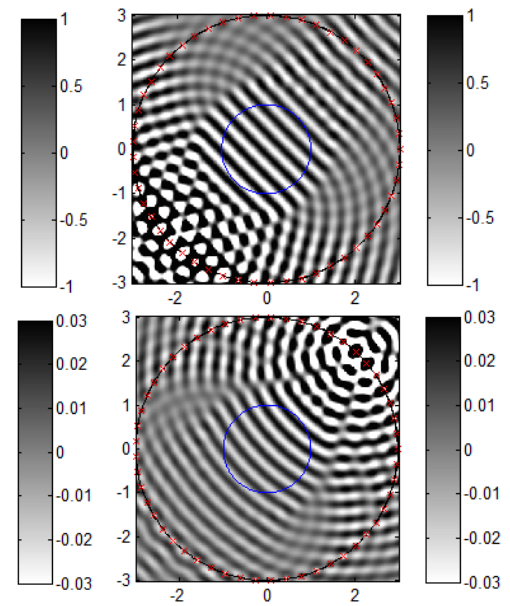

(c)

Fig. 2. Reproduction of a $2 \mathrm{D}$ plane wave from $\phi_{v}=\pi / 4$ (upper row) and a cylindrical wave from $\phi_{v}=\pi / 4, r_{v}=4 \mathrm{~m}$ (lower row) for frequency of $1 \mathrm{kHz}$. The loudspeakers are equally placed on a circle of radius $3 \mathrm{~m}$. The loudspeakers are shown as " $\mathrm{x}$ " marks: Desired sound field (a); 2.5D reproduction by the proposed method (b); 2.5D reproduction by the referenced method (c)

C) The third experiment: Additionally, in order to evaluate the reproduction performance of the proposed method for different frequency sound sources, the normalized reproduction error is also computed. The normalized reproduction error is defined as follows:

$$
\varepsilon_{M}(\boldsymbol{x}, k)=10 \log _{10} \frac{\int_{0}^{r_{r}} \int_{0}^{2 \pi}\left|P_{d}(\boldsymbol{x}, k)-P_{r}(\boldsymbol{x}, k)\right|^{2} r d \phi d r}{\int_{0}^{r_{r}} \int_{0}^{2 \pi}\left|P_{d}(\boldsymbol{x}, k)\right|^{2} r d \phi d r},
$$

where $r_{r}$ is the radius of the reproduction region, $k=2 \pi f / c ; P_{d}(\boldsymbol{x}, k)$ and $P_{r}(\boldsymbol{x}, k)$ represent the desired sound field and the reproduced sound field, respectively. According to (22), a more general representation of the reproduction performance is given in Fig. 3 where the normalized reproduction errors are shown as a function of frequency $f$.

In Fig. 3 the reproduction errors of the proposed method and the referenced method for a plane wave are represented. In these two methods, the radius of the reproduction region $r_{r}=3 \mathrm{~m}$, and according to $M=\left\lceil e k r_{r} / 2\right\rceil$, we can determine $M=76$. In the proposed method, the order of HO loudspeakers is 2, i.e., $\hat{N}=2$. Thus, $31 \mathrm{HO}$ loudspeakers are required in the proposed scheme. However, 153 point sources are used in the referenced method for 2.5D reproduction. In Fig. 3 it can be seen that the reproduction errors of the proposed method are close to those of 
the referenced method. However, the required number of loudspeakers in the referenced approach is approximately $2 \hat{N}+1$ times than that of the proposed method. This means that the proposed method results in a significant reduction in the number of loudspeakers. Furthermore, we note that the system designed at a particular frequency (i.e., $1 \mathrm{kHz}$ in the experiment), can provide accurate sound field reproduction performance for numbers fewer than the designed frequency. At large $f$ beyond the designed frequency, the reproduction errors become significant and monotonically increase with $f$, which validates the general principle [3] for choosing the required truncation order of (4).

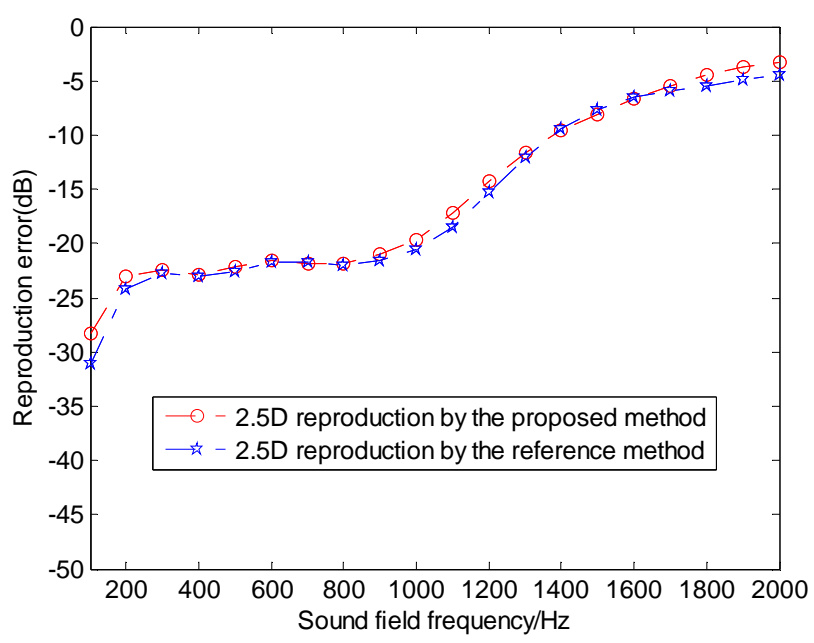

Fig. 3. Normalized reproduction error as a function of frequency $f$

\section{Conclusion}

In this paper, based on spherical harmonic expansions, we propose a method to reproduce $2 \mathrm{D}$ or height invariant sound fields with a circular array of $\mathrm{HO}$ loudspeakers. Based on the spherical addition theorem, a spherical harmonics representation of a 2D sound field reproduced by an array of HO loudspeakers is obtained. The modal weights of HO loudspeakers are designed by using the least squares method. Simulation results show that the proposed method extends the reproduction region and significantly reduces the minimum number of loudspeakers. Therefore, compared to the traditional methods, the implementation of the proposed method in commercial applications is more feasible and efficient.

Acknowledgments: This work has been supported by the National Natural Science Foundation of China (No 61231015, 61201197), Specialized Research Fund for the Doctoral Program of Higher Education of the Peoples Republic of China (No 20121103120017), the Scientific Research Project of Beijing Educational Committee (No KM201310005008) and the 13th Postgraduate Science Foundation of Beijing University of Technology (No ykj-2014-11552). 


\section{References}

1. Dolby Laboratories. Dolby Surround 7.1: Technical Information for Theaters. http://www.dolby.com/us/en/technologies/dolby-surround-7-1-for-theater-techpaper.pdf

2. Spors, S., R. Rabenste in, J. A hrens. The Theory of Wave Field Synthesis Revisited. In: Proc. of 124th Convention of the Audio Engineering Society, AES, Amsterdam, Netherlands, 2008, pp. 413-431.

3. W u, Y. J., T. D. A b h a y a a la. Theory and Design of Sound Field Reproduction Using Continuous Loudspeakers Concept. - IEEE Transactions on Audio, Speech, and Language Processing, Vol. 17, 2009, No 1, pp. 107-116.

4. W u, Y. J., T. D. A b h a y p a la. Spatial Multizone Soundfield Reproduction: Theory and Design. - IEEE Transactions on Audio, Speech, and Language Processing. Vol. 19, 2011, No 6, pp. 1711-1720.

5. Spors, S., J. A hrens. Analysis and Improvement of Pre-Equalization in 2.5-Dimensional Wave Field Synthesis. - In: Proc. of 128th Convention of the Audio Engineering Society, AES, London, UK, 2010, pp. 1789-1805.

6. Ahrens, J., S. Spors. Sound Field Reproduction Using Planar and Linear Arrays of Loudspeakers. - IEEE Transactions on Audio, Speech and Language Processing, Vol. 18, 2010, No 8, pp. 2038-2050.

7. A hre n s, J., S. S p or s. Applying the Ambisonics Approach to Planar and Linear Distributions of Secondary Sources and Combinations Thereof. - Acta Acustica United with Acustica, Vol. 98, 2012, No 1, pp. 28-36.

8. P o l e t t i, M. A. Three-Dimensional Surround Sound Systems Based on Spherical Harmonics. Journal of the Audio Engineering Society, Vol. 53, 2005, No 11, pp. 1004-1025.

9. Zhang, W., T. D. Abhayapala. 2.5D Sound Field Reproduction in Higher Order Ambisonics. - In: Proc. of 14th International Workshop on Acoustic Signal Enhancement, IEEE, Juan les Pins, France, 2014, pp. 342-346.

10. P o $1 \mathrm{e} \mathrm{t} \mathrm{t} \mathrm{i,} \mathrm{M.} \mathrm{A.,} \mathrm{T.} \mathrm{D.} \mathrm{A} \mathrm{b} \mathrm{h} \mathrm{a} \mathrm{y} \mathrm{a} \mathrm{p}$ a $\mathrm{l}$ a. Interior and Exterior Sound Field Control Using General Two-Dimensional First-Order Sources. - Journal of the Acoustical Society of America, Vol. 129, 2011, No 1, pp. 234-244.

11. Poletti, M. A., T. D. A b hay a a la. Spatial Sound Reproduction Systems Using Higher Order Loudspeakers. - In: Proc. of IEEE International Conference on Acoustics, Speech and Signal Processing, IEEE, Prague, Czech Republic, 2011, pp. 57-60.

12. S a ma r a sing he, P. N., M. A. P o letti, T. D. A b h a y a p l a. 3D Soundfield Reproduction Using Higher Order Loudspeakers. - In: Proc. of IEEE International Conference on Acoustics, Speech and Signal Processing, Vancouver, Canada, 2013, pp. 306-310.

13. Dura is wa mi, R., D. Z otkin. High Order Spatial Audio Capture and Its Binaural HeadTracked Playback over Headphones with HRTF Cues. - In: Proc. of 119th Convention of the Audio Engineering Society, AES, New York, USA, 2005, pp. 1186-1201.

14. W a r d, D. B., T. D. A b h a y a p a 1 a. Reproduction of Plane Wave Sound Field Using an Array of Loudspeakers. - IEEE Transactions on Speech Audio Process, Vol. 9, 2001, No 6, pp. 697-707.

15. Poletti, M. A., T. B e $\mathrm{l}$ e h e m, T. D. A b h a y a p a la. Comparison of Sound Reproduction Using Higher Order Loudspeakers and Equivalent Line Arrays in Free-Field Conditions. Journal of the Acoustical Society of America, Vol. 136, 2014, No 1, pp. 192-200.

16. S a mara inghe, P. N., T. D. Abh a y pala, M. A. Poletti. 3D Spatial Sound Field Recording over Large Regions. - In: Proc. of 12th International Workshop on Acoustic Signal Enhancement (IWAENC), IEEE, Aachen, Germany, 2012, pp. 1-4.

17. A hrens, J., S. S p ors. An Analytical Approach to Sound Field Reproduction Using Circular and Spherical Loudspeaker Distributions. - Acta Acustica United with Acustica, Vol. 94, 2008, No 6, pp. 988-999.

18. S u n, H., S. Y a n, U. P. S ven s s o n. Optimal Higher Order Ambisonics Encoding with Predefined Constraints. - IEEE Transactions on Audio, Speech, and Language Processing, Vol. 20, 2011, No 3, pp. 742-754. 